
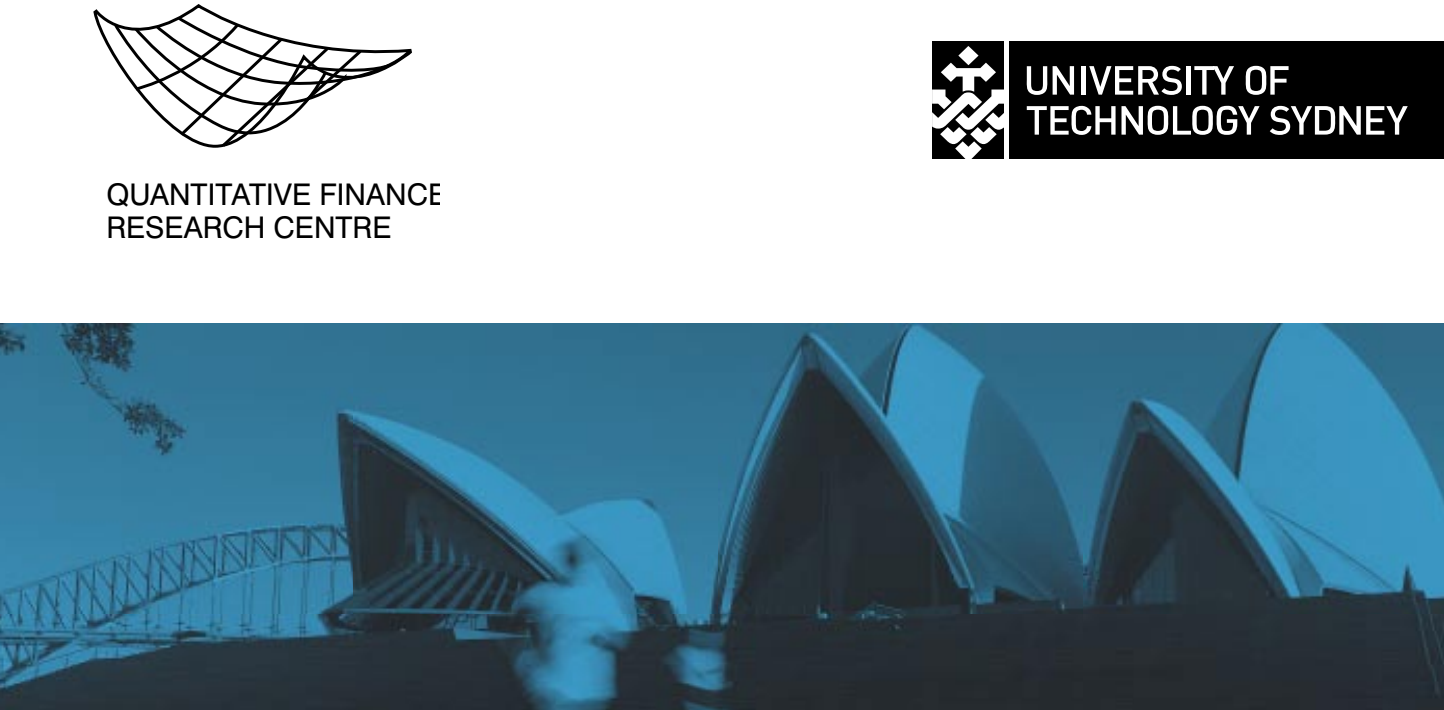 \\ NCE
}
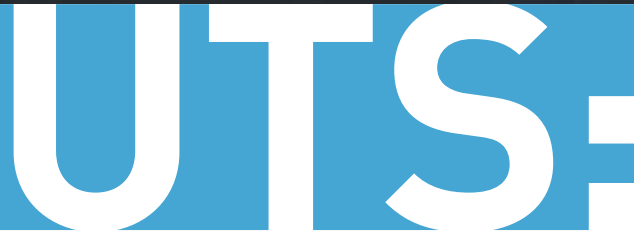

THINK.CHANGE.DO

\section{Quantitative Finance Research Centre}

Research Paper 171

November 2005

The Impact of Short-Sale Constraints on Asset Allocation Strategies via the Backward Markov Chain Approximation Method

Carl Chiarella and Chih-Ying Hsiao 


\title{
The Impact of Short-Sale Constraints on Asset Allocation Strategies via the Backward Markov Chain Approximation Method
}

\author{
Carl Chiarella* Chih-Ying Hsiao ${ }^{\dagger}$
}

7 November, 2005

\begin{abstract}
This paper considers an asset allocation strategy over a finite period under investment uncertainty and short-sale constraints as a continuoustime stochastic control problem. Investment uncertainty is characterised by a stochastic interest rate and inflation risk. If there are no short-sale constraints, the optimal asset allocation strategy can be solved analytically. We consider several kinds of short-sale constraints and employ the backward Markov chain approximation method to explore the impact of short-sale constraints on asset allocation decisions. Our results show that the short-sale constraints do indeed have a significant impact on the asset allocation decisions.
\end{abstract}

\section{Introduction}

Recently, continuous-time stochastic control/optimization methods have found many applications in modern finance research. This paper considers a problem where asset allocation strategies - including both consumption and portfolio strategies, are constructed optimally in an intertemporal and stochastic framework. In particular, we are interested in the impact of short-sale constraints on asset allocation strategies under stochastic interest rate and inflation risks.

In Merton's $(1971,1973)$ seminal works, the method of dynamic programming was applied to solve for such intertemporal asset allocation strategies. Using this technique, Merton showed the existence of an intertemporal hedging term which does not appear in a myopic strategy in a frictionless market. He solved the Hamiliton-Jacobi-Bellman equation analytically for special examples. Liu(2005)

\footnotetext{
* School of Finance and Economics, University of Technology, Sydney, Australia

${ }^{\dagger}$ University of Bielefeld, Germany
} 
has extended the class of solvable examples of problems of the type considered by Merton to a much broader family. Cox and Huang (1989) develop an alternative method to dynamic programming to solve intertemporal asset allocation problems in a frictionless and complete market. In certain situations their static variational method facilitates the solvability for such problems; see for example, Wachter (2002).

In addition to uncertainty of asset returns, inflation risk is another important factor that needs to be taken into consideration when constructing long-term asset allocation strategies since the main purpose of investment in financial markets is usually to have a consumptions bundle at a later time. For this reason, our framework extends that of Merton and those above by considering a stochastic price level which casts additional risk on consumption decisions. In order to hedge such inflation risk, agents in our model can invest in inflation-indexed bonds which give a fixed payout in units of consumption goods at a future date. The pricing dynamics and empirical behavior of such bonds are discussed in detail in Hsiao, Chiarella and Semmler (2005).

In order to account for the real-world trading environment we also allow for short-sale constraints. The intertemporal control problem can be readily handled when short-sale constraints are considered. However it turns out that to solve the intertemporal control problem in this case one has to resort to computational methods. Tapiero and Sulem (1994) summarize such computational methods into four categories: (i) methods for solving the discretised HamiltonJacobi-Bellman (HJB) equation, (ii) the Markov chain approximation method of Kushner (1977) which approximates the original controlled process by finitestate processes, (iii) methods for such examples with well-known solutions, and (iv) methods using simulation-based techniques. In this paper we employ the Markov chain approximation method and develop a backward iteration scheme to solve our finite-period stochastic control problem. We will discuss the convergence of the backward (Markov chain) approximation method. Although formally, it is difficult to prove the convergence rigorously we do observe convergence in the numerical examples we consider.

The structure of this paper is as follows. In Section 2 we introduce our asset allocation model with a stochastic price level and with investment in inflationindexed bonds. The analytical solution without short-sale constraints is also provided. In Section 3 we present the algorithm of the backward approximation method and consider the convergence conditions. Section 4 contains a numerical study where the backward approximation method is applied for the case with and without short-sale constraints. Finally, Section 5 concludes the paper. 


\section{The Intertemporal Model}

\subsection{Optimal Intertemporal Asset Allocation as a Stochas- tic Control Problem}

We consider the following continuous-time stochastic control problem. There are identical agents who have wealth endowment $V_{0}>0$ at initial time and maximize their life-time expected utility ${ }^{1}$

$$
J\left(0, T, V_{0}, X_{0}\right)=\max _{\alpha_{t}, c_{t} ; t \in[0, T]} \mathbf{E}_{0}\left[\epsilon_{1} \int_{0}^{T} e^{-\delta t} U\left(c_{t}\right) d t+e^{-\delta T} U\left(c_{T}\right)\right],
$$

by deciding their real consumption $c_{t}$ and investment proportions $\alpha_{t}$ over the horizon $[0, T]$. The utility at time $t$ is a function of the real consumption $c_{t}$ and is discounted by the factor $e^{-\delta t}$. The utility function $U$ is time-invariant and is of the constant relative risk aversion (CRRA) type

$$
U\left(c_{t}\right)=\frac{c_{t}^{1-\gamma}}{1-\gamma}, \quad \text { with } \gamma>0
$$

With $\epsilon_{1}=1$ all intermediate consumption is taken into consideration while with $\epsilon_{1}=0$ only final expected utility is considered.

The agents can invest in a financial market consisting of $m$ risky assets and one risk-less asset (money market account). We use $P_{o t}$ and $P_{i t}$ to denote respectively the prices of the risk-less asset and risky asset $i \quad(i=1, \cdots, n)$. The (instantaneous) return of the risk-less asset is the nominal instantaneous interest rate $R_{t}$, so that

$$
\frac{d P_{0 t}}{P_{0 t}}=R_{t} d t
$$

and the return of the $m$ risky assets are described by the $m$-dimensional diffusion process

$$
\frac{d P_{i t}}{P_{i t}}=\mu_{i}\left(X_{t}, t\right) d t+\Sigma_{i}\left(X_{t}, t\right) d W_{t}, \quad \text { for } i=1, \cdots, m
$$

where $\mu_{i}$ are $\mathbb{R}^{n} \times \mathbb{R}_{+} \rightarrow \mathbb{R}$ functions, $\Sigma_{i}$ are $\mathbb{R}^{n} \times \mathbb{R}_{+} \rightarrow \mathbb{R}^{1 \times m}$, and $W_{t}=$ $\left(W_{1 t}, \cdots, W_{m t}\right)^{\top}$ is an $m$-dimensional Wiener process with the correlation coefficients $\mathcal{R}_{A A} d t:=d W_{t} d W_{t}^{\top}$.

The instantaneous nominal interest rate $R_{t}$, the drift coefficients $\mu_{i}$ and the diffusion coefficients $\Sigma_{i}$ are assumed to be functions of $n$ underlying factors $X_{t}=\left\{X_{1}, \cdots X_{n}\right\}$ whose dynamics are given exogenously by the $n$-dimensional diffusion process

\footnotetext{
${ }^{1}$ The arguments of the life-time expected utility $J$ will be explained below.
} 


$$
d X_{t}=F\left(X_{t}\right) d t+G\left(X_{t}\right) d W_{t}^{X}
$$

where $X_{t}=\left(X_{1 t}, \cdots, X_{n t}\right)^{\top}, F: \mathbb{R}^{n \times 1} \rightarrow \mathbb{R}^{n \times 1}, G: \mathbb{R}^{n \times 1} \rightarrow \mathbb{R}^{n \times n} . W_{t}^{X}=$ $\left(W_{1 t}^{X}, \cdots, W_{n t}^{X}\right)^{\top}$ is an $n$-dimensional Wiener process with the correlation matrix $\mathcal{R}_{X X} d t:=d W_{t}^{X} d W_{t}^{X \top}$. The shocks $W_{t}^{X}$ and $W_{t}$ are correlated with the correlation coefficient $\mathcal{R}_{X A}$. Examples of such factors are interest rates, expected inflation, stochastic drifts, stochastic volatilities, and Sharpe ratio amongst others. We assume the factor $X_{t}$ has a stationary distribution.

The investment decision is denoted by investment proportions $\alpha_{i t}$ of investor wealth in the $i$-th asset with $\sum_{i=0}^{m} \alpha_{i t} \equiv 1$ for all $t \in[0, T]$. Inflation risk is taken into account and is characterized by a stochastic price index $I_{t}$ described by

$$
\frac{d I_{t}}{I_{t}}=\pi_{t} d t+\sigma_{I} d W_{t}^{I}
$$

where $W_{t}^{I}$ is a one-dimensional Wiener process. Here $\pi_{t}$ is the anticipated instantaneous inflation rate, which is assumed to be a function of the factors $\pi_{t}=\pi\left(X_{t}\right)$. The correlation matrices between the shocks are denoted by $\mathcal{R}_{I X} d t:=d W_{t}^{I} d W_{t}^{X}$ and $\mathcal{R}_{I A} d t:=d W_{t}^{I} d W_{t}$. We normalize the inflation index by setting $I_{0} \equiv 1$.

Let $V_{t}$ denote agents' nominal wealth at time $t$. Given decisions of investment proportions $\alpha_{t}:=\left(\alpha_{1 t}, \cdots, \alpha_{n t}\right)^{\top}$ and nominal consumption $C_{t}$, agents' nominal wealth changes at the rate

$$
\frac{d V_{t}}{V_{t}}=\left(R_{t}-\psi_{t}\right) d t+\alpha_{t}^{\top}\left(\left(\mu_{t}-R_{t} \underline{\mathbf{1}}\right) d t+\Sigma_{t} d W_{t}\right),
$$

where $\underline{\mathbf{1}}=(1, \cdots, 1)^{\top}, \psi_{t}:=C_{t} / V_{t}$ is the nominal consumption ratio, and

$$
\mu_{t}=\left(\begin{array}{c}
\mu_{1}\left(X_{t}, t\right) \\
\vdots \\
\mu_{n}\left(X_{t}, t\right)
\end{array}\right) \quad \text { and } \quad \Sigma_{t}=\left(\begin{array}{c}
\Sigma_{1}\left(X_{t}, t\right) \\
\vdots \\
\Sigma_{n}\left(X_{t}, t\right)
\end{array}\right)
$$

Since agents' utility is a function of real consumption we need to derive the dynamics of real wealth $v_{t}:=V_{t} / I_{t}$. Applying Itô's Lemma to (6) and making use of the dynamics of nominal wealth (7) we can obtain the dynamics of real wealth

$$
\begin{aligned}
\frac{d v_{t}}{v_{t}}= & d\left(\frac{V_{t}}{I_{t}}\right) \frac{I_{t}}{V_{t}}=\left[\left(R_{t}-\psi_{t}-\pi_{t}+\sigma_{I}^{2}\right)\right. \\
& \left.+\alpha_{t}^{\top}\left(\mu_{t}-R_{t} \underline{\mathbf{1}}-\sigma_{I} \Sigma_{t} \mathcal{R}_{I}\right)\right] d t+\alpha^{\top} \Sigma_{t} d W_{t}-\sigma_{I} d W_{t}^{I}
\end{aligned}
$$


When we consider short-sale constraints, for example, an additional short-sale commission $\eta$ for each unit of short position, then the equation for real wealth dynamics becomes

$$
\begin{aligned}
\frac{d v_{t}}{v_{t}}= & {\left[\left(R_{t}-\psi_{t}-\pi_{t}+\sigma_{I}^{2}\right)+\sum_{i=0}^{m} \min \left(0, \alpha_{i t}\right) \eta\right.} \\
& \left.+\alpha_{t}^{\top}\left(\mu_{t}-R_{t} \underline{\mathbf{1}}-\sigma_{I} \Sigma_{t} \mathcal{R}_{I}\right)\right] d t+\alpha^{\top} \Sigma_{t} d W_{t}-\sigma_{I} d W_{t}^{I} .
\end{aligned}
$$

In summary, our stochastic control problem is to maximizes the life-time expected utility (1) by choosing $\left\{c_{t}, \alpha_{t}\right\}$ for all $t \in[0, T]$. The state variables are $\left\{X_{t}, v_{t}\right\}$ with the dynamics (5), and (8) or (9). We note that our control problem is non-autonomous because bonds will be included as assets and their expected returns will vary stochastically with time to maturity.

\subsection{The HJB Equation and Reformulation}

First we briefly review the Hamilton-Jacobi-Bellman (HJB) equation. Let $J\left(t, T, v_{t}, X_{t}\right)$ denote the optimized objective function (value function) over the sub-period $[t, T]$ with the given initial real wealth $v_{t}$ and the given state of the factor $X_{t}$, so that

$$
J\left(t, T, v_{t}, X_{t}\right)=\max _{\psi_{s}, \alpha_{s} ; t \leq s \leq T}\left\{\mathbf{E}_{t}\left[\epsilon_{1} \int_{t}^{T} e^{-\delta s} U\left(\psi_{s} v_{s}\right) d s+e^{-\delta T} U\left(v_{T}\right)\right]\right\} .
$$

The Hamilton-Jacobi-Bellman(HJB) equation ${ }^{2}$ characterizes the first order conditions for the value function, namely

$$
\begin{aligned}
& 0=\max _{\psi_{t}, \alpha_{t}}\left\{\epsilon_{1} e^{-\delta t} U\left(\psi_{t} v_{t}\right)+\frac{\partial}{\partial t} J\right. \\
& +\left[R_{t}-\psi_{t}-\pi_{t}+\sigma_{I}^{2}+\alpha_{t}^{\top}\left(\mu_{t}-R_{t} \underline{\mathbf{1}}-\Sigma_{t} \mathcal{R}_{I} \sigma_{I}\right)\right. \\
& \left.+\left(\sum_{i=0}^{m} \min \left(0, \alpha_{i t}\right) \eta\right)\right] J_{v} v_{t} \\
& +\frac{1}{2}\left(\alpha_{t}^{\top} \Sigma_{t} \mathcal{R}_{A A} \Sigma_{t}^{\top} \alpha_{t}-2 \sigma_{I} \alpha_{t}^{\top} \Sigma_{t} \mathcal{R}_{A I}+\sigma_{I}^{2}\right) J_{v v} v_{t}^{2} \\
& +\left(\alpha_{t}^{\top} \Sigma_{t} \mathcal{R}_{A X} \Sigma_{t}^{X \top}-\sigma_{I} \mathcal{R}_{I} \Sigma_{t}^{X \top}\right) J_{v X} v_{t} \\
& \left.+F_{t}^{\top} J_{X}+\frac{1}{2} \sum_{i, j=1}^{n} G_{i t} \mathcal{R}_{X X} G_{j t}^{X \top} J_{X_{i} X_{j}}\right\} .
\end{aligned}
$$

\footnotetext{
${ }^{2}$ The intuition for the HJB equation lies in the infinitesimal decomposition

$$
J\left(t, T, v_{t}, X_{t}\right)=\max _{\psi_{s}, \alpha_{s} ; t \leq s \leq T}\left\{e^{-\delta t} U\left(\psi_{t} v_{t}\right) d t+J\left(t+d t, T, v_{t+d t}, V_{t+d t}\right)\right\} .
$$

See P.264-271 in Kamien and Schwartz (1991) for a heuristic discussion and Chapter 11 in $\varnothing \mathrm{ksendal}(2000)$ for a rigorous derivation.
} 
For the case without short-sale commissions we have simply $\eta \equiv 0$.

We observe that the transition dynamics of the state variables $v_{t}$ (equation 8) and $X_{t}$ (equation 5) are independent of the (real) wealth level. Together with the homothecity of the utility function, the optimal controls $\psi_{t}$ and $\alpha_{t}$ turn out to be independent of the real wealth level $v_{t}$. Therefore, we can decompose the value function into a product form, namely

$$
\begin{aligned}
& J\left(t, T, v_{t}, X_{t}\right) \\
= & v_{t}^{1-\gamma} \max _{\psi_{s}, \alpha_{s} ; t \leq s \leq T}\left\{\epsilon_{1} \mathbf{E}_{t}\left[\int_{t}^{T} e^{-\delta s} U\left(\psi_{s} \frac{v_{s}}{v_{t}}\right) d s\right]+e^{-\delta T} \mathbf{E}_{t}\left[U\left(\frac{v_{T}}{v_{t}}\right)\right]\right\} \\
= & v_{t}^{1-\gamma} J\left(t, T, 1, X_{t}\right) \\
=: & e^{-\delta t} U\left(v_{t}\right) \Phi\left(t, T, X_{t}\right)^{\gamma},
\end{aligned}
$$

where

$$
\Phi\left(t, T, X_{t}\right)=\left(e^{\delta t}(1-\gamma) J\left(t, T, 1, X_{t}\right)\right)^{\frac{1}{\gamma}}
$$

is not a function of $v_{t}$. From the HJB equation (11) we obtain the first order condition for $\psi_{t}$ and $\alpha_{t}$. We have, for the case $\epsilon_{1}=1$,

$$
\psi_{t}^{*}=\frac{1}{\Phi\left(t, T, X_{t}\right)} .
$$

For the case without intermediate consumption $\left(\epsilon_{1}=0\right)$ the optimal consumption decision is $\psi_{t}^{*}=0$.

In the case without any short-sale constraints, the optimal portfolio $\alpha_{t}$ can be solved as

$$
\begin{aligned}
\alpha_{t}^{*}=\left(\Sigma_{t} \mathcal{R}_{A A} \Sigma_{t}^{\top}\right)^{-1} & \left(-\frac{J_{v} v_{t}}{J_{v v} v_{t}^{2}}\left(\mu_{t}-R_{t} \underline{\mathbf{1}}\right)-\frac{1}{J_{v v} v_{t}^{2}} \Sigma_{t} \mathcal{R}_{A X} \Sigma_{t}^{X \top} J_{v X} v_{t}\right. \\
& \left.+\frac{J_{v} v_{t}+J_{v v} v_{t}^{2}}{J_{v v} v_{t}^{2}} \sigma_{I} \Sigma_{t} \mathcal{R}_{A I}\right) .
\end{aligned}
$$

In the more general situation with short-sale restrictions (for example, the shortsale commission fee $\eta>0$ in the HJB equation (11)) we are not able to solve for $\alpha_{t}^{*}$ explicitly, so we will employ the computational algorithm introduced later to give a numerical solution.

For the remainder of this subsection we consider only the case without any short-sale constraints. Applying the expressions (13), (14) and the product form (12) to the HJB equation (11), the HJB equation is transformed into a partial differential equation for the function $\Phi$, namely 


$$
\begin{aligned}
& \quad 0=\epsilon_{1}+\frac{\partial}{\partial t} \Phi+F_{t}^{\top} \Phi_{X} \\
& +\quad\left(\frac{1-\gamma}{\gamma} G_{t} \mathcal{R}_{X A} \mathcal{R}_{A A}^{-1} \Sigma_{t}^{-1}\left(\mu_{t}-R_{t} \underline{\mathbf{1}}\right)-\frac{(1-\gamma)^{2}}{\gamma} G_{t} \mathcal{R}_{X A} \mathcal{R}_{A A}^{-1} \mathcal{R}_{A I} \sigma_{I}-(1-\gamma) G_{t} \mathcal{R}_{X I} \sigma_{I}\right)^{\top} \Phi_{X} \\
& +\frac{1}{2} \sum_{i, j=1}^{n} \Phi_{X_{i} X_{j}} G_{i t} \mathcal{R}_{X X} \Sigma_{j t}^{X \top} \\
& +\frac{1-\gamma}{2 \Phi} \sum_{i, j=1}^{n} \Phi_{X_{i}} \Phi_{X_{j}}\left(G_{i t} \mathcal{R}_{X A} \mathcal{R}_{A A}^{-1} \mathcal{R}_{A X} G_{j t}^{\top}-G_{i t} \mathcal{R}_{X X} G_{j t}^{\top}\right) \\
& +\quad \Phi\left(-\frac{\delta}{\gamma}+\frac{1-\gamma}{\gamma}\left(R_{t}-\pi_{t}+\sigma_{I}^{2}\right)+\frac{1-\gamma}{2 \gamma^{2}}\left(\mu_{t}-R_{t} \underline{\mathbf{1}}\right)^{\top}\left(\Sigma_{t} \mathcal{R}_{A A} \Sigma_{t}^{\top}\right)^{-1}\left(\mu-R_{t} \underline{\mathbf{1}}\right)\right. \\
& \left.\quad+\frac{(1-\gamma)^{3}}{2 \gamma^{2}} \sigma_{I}^{2} \mathcal{R}_{I A} \mathcal{R}_{A A}^{-1} \mathcal{R}_{A I}-\frac{(1-\gamma)^{2}}{\gamma^{2}}\left(\mu_{t}-R_{t}\right)^{\top} \Sigma_{t}^{\top-1} \mathcal{R}_{A A}^{-1} \mathcal{R}_{A I} \sigma_{I}-\frac{1-\gamma}{2} \sigma_{I}^{2}\right)
\end{aligned}
$$

The main issue then is to solve the partial differential equation (15). In the next section we will specify the assets in our model and show that the partial differential equation above can be solved analytically under the assumed asset specification. In the case with short-sale constraints, we are not able to solve the corresponding partial differential equation analytically and we need to employ a computational algorithm to obtain a numerical solution, as shown later in Section 4.

\section{$2.3 \quad$ Specification of Assets}

The previous subsection introduced a general model of asset allocation, now we will specify our universe of investment assets more precisely. Our set of risky assets includes (zero-coupon) nominal bonds, Inflation-Indexed Bonds (IIB) and stocks. The payout of an inflation-indexed bond is adjusted to a current price index, for example, the inflation-indexed bonds issued by the US Treasury since 1997 - Treasury Inflation-Protected Securities - are adjusted to the Consumer Price Index.

The risk factors are the instantaneous real interest rate $r_{t}$ and the instantaneous expected inflation $\pi_{t}$ described by the processes

$$
d r_{t}=\kappa_{r}\left(\bar{r}-r_{t}\right) d t+g_{r} d W_{t}^{r},
$$

and

$$
d \pi_{t}=\kappa_{\pi}\left(\bar{\pi}-\pi_{t}\right) d t+g_{\pi} d W_{t}^{\pi} .
$$

where $W_{t}^{r}$ and $W_{t}^{\pi}$ are correlated Wiener processes with the instantaneous correlation $d W_{t}^{r} d W_{t}^{\pi}=\rho_{r \pi} d t$. 
The price of a (zero-coupon) nominal bond at time $t$ is denoted by $P\left(r_{t}, \pi_{t}, t, T\right)$. At the maturity date $T$ the bond holder obtains one unit of money. We shall assume that this price can be modelled by a two-factor exponential affine model of the Duffie and Kan (1996) type, so that the bond price has the form ${ }^{3}$

$$
P_{n}\left(r_{t}, \pi_{t}, t, T\right)=\exp \left(-A_{n}(T-t)-B_{n r}(T-t) r_{t}-B_{n \pi}(T-t) \pi_{t}\right) .
$$

The coefficient $B_{n r}(T-t)$ is the elasticity that measures the relative response of the nominal bond price with respect to the change of $r_{t}$. The bond return and the instantaneous nominal interest rate satisfy the relation

$$
R_{t}:=\lim _{T \downarrow t} \frac{-\ln P_{n}\left(r_{t}, \pi_{t}, t, T\right)}{T-t}=A_{n}^{\prime}(0)+B_{n r}^{\prime}(0) r_{t}+B_{n \pi}^{\prime}(0) \pi_{t}
$$

The value of the risk-less money market account has the form

$$
P_{0 t}=\exp \left(\int_{0}^{t} R_{s} d s\right) .
$$

The price of a (zero-coupon) inflation-indexed bond (IIB) at $t$ is denoted by $P_{I}(t, T)$. At the maturity date $T$ the bond holders obtain the nominal value amounting to one unit of consumption good which is equal to $I_{T}$. Normalizing the IIB with respect to the price index $P_{I}(t, T) / I_{t}=: P_{r}(t, T)$ we obtain a real bond valued in units of the consumption good. We assume that the real bond price depends only on the factor $r_{t}$ and that it can also be modelled by the exponential affine family according to

$$
P_{r}\left(r_{t}, t, T\right)=\exp \left(-A_{r}(T-t)-B_{r r}(T-t) r_{t}\right) .
$$

With respect to the real interest rate we can construct a consumption good account $M_{r}(t)$ defined as an accumulation of the instantaneous "goods" return

$$
M_{r}(t)=\exp \left(\int_{0}^{t} r_{s} d s\right) .
$$

Let the real money account $M_{I}(t)$ represent the nominal value of the consumption good account,

$$
M_{I}(t)=M_{r}(t) I_{t} .
$$

The key assumption for our bond market is the no-arbitrage constraint. We follow the setting of Jarrow and Yildirim (2003) where any arbitrage opportunity is excluded by constructing bond portfolios including inflation-indexed bonds.

Proposition 1 The discounted nominal bond prices $P_{n}(t, T) / P_{0 t}$, the discounted prices of the inflation-indexed bonds $P_{I}(t, T) / P_{0 t}$ for $t \in[0, T]$ with arbitrary maturity dates $T>0$ and the discounted real money account $M_{I}(t) / P_{0 t}$ are martingales w.r.t. a risk-neutral measure.

\footnotetext{
${ }^{3}$ See the appendix for the expressions $A_{n}, B_{n r}, B_{n \pi}$.
} 
For the proof see Jarrow and Yildirim (2003).

With the above assumptions and by use of the rules of stochastic calculus, the following results obtain as a consequence of no-arbitrage:

Proposition 2 (i) The return process for the nominal bond is given by

$$
\begin{aligned}
& \frac{d P_{n}\left(r_{t}, \pi_{t}, t, T\right)}{P_{n}\left(r_{t}, \pi_{t}, t, T\right)}=\mu_{n}(t, T-t) d t-B_{n r}(T-t) g_{r} d W_{t}^{r}-B_{n \pi}(T-t) g_{\pi} d W_{t}^{\pi} \\
& \text { with } \\
& \qquad \begin{array}{c}
\mu_{n}(t, T-t)=R_{t}+\lambda_{r}\left(-g_{r} B_{n r}(T-t)\right)+\lambda_{\pi}\left(-g_{\pi} B_{n \pi}(T-t)\right) \\
\qquad B_{n r}(\tau)=\frac{1}{\kappa_{r}}\left(1-e^{-\kappa_{r} \tau}\right), \\
B_{n \pi}(\tau)=\frac{1}{\kappa_{\pi}}\left(1-e^{-\kappa_{\pi} \tau}\right) .
\end{array}
\end{aligned}
$$

(ii) The return process for the inflation-index bonds is given by

$$
\frac{d P_{I}\left(r_{t}, I_{t}, t, T\right)}{P_{I}\left(r_{t}, I_{t}, t, T\right)}=\mu_{I}(t, T-t) d t-B_{r r}(T-t) g_{r} d W_{t}^{r}+\sigma_{I} W_{t}^{I},
$$

with

$$
\begin{gathered}
\mu_{I}(t, T-t)=R_{t}+\lambda_{r}\left(-g_{r} B_{r r}(T-t)\right)+\lambda_{I} \sigma_{I}, \\
B_{r r}(\tau)=\frac{1}{\kappa_{r}}\left(1-e^{-\kappa_{r} \tau}\right) .
\end{gathered}
$$

(iii) The real interest rate, nominal interest rate and rate of inflation are related by

$$
\pi_{t}+r_{t}-R_{t}=\lambda_{I} \sigma_{I} .
$$

For details of the proof see See Hsiao, Chiarella and Semmler (2005) .

The constants $\lambda_{r}, \lambda_{\pi}$ and $\lambda_{I}$ are the market prices of risk and represent the excess return per unit risk of $W_{t}^{r}, W_{t}^{\pi}$ and $W_{t}^{I}$ respectively.

The stock price in our model is assumed to have both constant excess return and volatility and so is modelled by

$$
\frac{d P_{S}(t)}{P_{S}(t)}=\left(R_{t}+\lambda_{S} \sigma_{S}\right) d t+\sigma_{S} d W_{t}^{S},
$$

where $\sigma_{S}, \lambda_{S}$ (the market price of stock market risk) are positive constants.

We now put five assets into our investment set: the nominal money account 
$P_{0 t}$, two nominal bonds with different maturities $T_{1}, T_{2}$, one IIB maturing at $T_{3}$ and one stock. Their returns are summarized in vector form as

$$
\begin{aligned}
\frac{d P_{0 t}}{P_{0 t}} & =R_{t} d t \\
\left(\begin{array}{c}
d P_{n}\left(t, T_{1}\right) / P_{n}\left(t, T_{1}\right) \\
d P_{n}\left(t, T_{2}\right) / P_{n}\left(t, T_{2}\right) \\
d P_{I}\left(t, T_{3}\right) / P_{I}\left(t, T_{3}\right) \\
d P_{S}(t) / P_{S}(t)
\end{array}\right) & =\mu_{t} d t+\Sigma_{t} d W_{t},
\end{aligned}
$$

where $d W_{t}=\left(d W_{t}^{r}, d W_{t}^{\pi}, d W_{t}^{I}, d W_{t}^{S}\right)^{\top}$,

$$
\begin{aligned}
\Sigma_{t} & =\left(\begin{array}{cccc}
-B_{n r}\left(T_{1}-t\right) g_{r} & -B_{n \pi}\left(T_{1}-t\right) g_{\pi} & 0 & 0 \\
-B_{n r}\left(T_{2}-t\right) g_{r} & -B_{n \pi}\left(T_{2}-t\right) g_{\pi} & 0 & 0 \\
-B_{r r}\left(T_{3}-t\right) g_{r} & 0 & \sigma_{I} & 0 \\
0 & 0 & 0 & \sigma_{S}
\end{array}\right), \\
\mu_{t} & =R_{t} \underline{\mathbf{1}}+\Sigma_{t} \lambda,
\end{aligned}
$$

with $\underline{\mathbf{1}}=(11111)^{\top}$ and $\lambda=\left(\lambda_{r} \lambda_{\pi} \lambda_{I} \lambda_{S}\right)^{\top}$. Recall the factors $X$, the dynamics of which are given by (5), affect the drifts and diffusions of the return processes. Here the factors relevant to the asset specification above are the real interest rate and inflation rate so that $X_{t}=\left(r_{t}, \pi_{t}\right)$. As far as the investment uncertainty is concerned we have four sources of return risks namely, $W_{t}^{r}, W_{t}^{\pi}, W_{t}^{I}, W_{t}^{S}$.

\subsection{Analytical Solution in the case without Trade Restric- tions}

In this subsection we will solve the partial differential equation (15) (the case of no short-sale constraints) for the assets specified in Section 2.3. We note at first that the factor uncertainty $W_{t}^{X}=\left(W_{t}^{r}, W_{t}^{\pi}\right)^{\top}$ is a subset of the return uncertainty $W_{t}=\left(W_{t}^{r}, W_{t}^{\pi}, W_{t}^{I}, W_{t}^{S}\right)^{\top}$, so that

$$
\mathcal{R}_{X A} \mathcal{R}_{A A}^{-1} \mathcal{R}_{A X}=\mathcal{R}_{X X}
$$

and the fourth line of the equation (15) then vanishes. The equation (15) reduces to a form which can be solved by the Feynman-Kac Formula. Also, we use the 
no-arbitrage equality (31) to simply the equation (15) to obtain

$$
\begin{aligned}
& 0=\epsilon_{1}+\frac{\partial}{\partial t} \Phi+F_{t}^{\top} \Phi_{X} \\
& +\quad\left(\frac{1-\gamma}{\gamma} G_{t} \mathcal{R}_{X A} \mathcal{R}_{A A}^{-1} \lambda-\frac{(1-\gamma)^{2}}{\gamma} G_{t} \mathcal{R}_{X A} \mathcal{R}_{A A}^{-1} \mathcal{R}_{A I} \sigma_{I}-(1-\gamma) G_{t} \mathcal{R}_{X I} \sigma_{I}\right)^{\top} \Phi_{X} \\
& +\quad \frac{1}{2} \sum_{i, j=1}^{n} \Phi_{X_{i} X_{j}} G_{i t} \mathcal{R}_{X X} \Sigma_{j t}^{X \top} \\
& +\quad \Phi\left(-\frac{\delta}{\gamma}+\frac{1-\gamma}{\gamma}\left(R_{t}-\pi_{t}+\sigma_{I}^{2}\right)+\frac{1-\gamma}{2 \gamma^{2}} \lambda^{\top} \mathcal{R}_{A A}^{-1} \lambda\right. \\
& \left.\quad+\frac{(1-\gamma)^{3}}{2 \gamma^{2}} \sigma_{I}^{2} \mathcal{R}_{I A} \mathcal{R}_{A A}^{-1} \mathcal{R}_{A I}-\frac{(1-\gamma)^{2}}{\gamma^{2}} \lambda^{\top} \mathcal{R}_{A A}^{-1} \mathcal{R}_{A I} \sigma_{I}-\frac{1-\gamma}{2} \sigma_{I}^{2}\right)
\end{aligned}
$$

The solution details can be found in Hsiao, Chiarella and Semmler (2005) and the main result can be summarized as:

Proposition 3 (i) the value function $\Phi\left(t, T, X_{t}\right)$ is given by

$$
\begin{aligned}
& \Phi\left(t, T, X_{t}\right) \\
= & \exp \left(\frac{1-\gamma}{\gamma} B_{r}(t, T) r_{t}+h(T-t)+\frac{1-\gamma}{\gamma}\left(T-t-B_{r}(t, T)\right)\left(\bar{r}+\frac{1-\gamma}{\gamma} \frac{g_{r}^{2}}{2 \kappa_{r}}+\hat{z}_{1} \frac{g_{r}}{\kappa_{r}}\right)\right)
\end{aligned}
$$

where

$$
\begin{aligned}
z & =\frac{1-\gamma}{\gamma}\left(\begin{array}{c}
\lambda_{r}-\sigma_{I} \rho_{I r} \\
\lambda_{\pi}-\sigma_{I} \rho_{I \pi}
\end{array}\right) \\
h & =-\frac{\delta}{\gamma}+\frac{1-\gamma}{2 \gamma^{2}} \lambda^{\top} \mathcal{R}_{A A}^{-1} \lambda+\frac{(1-\gamma) \sigma_{I}^{2}}{2 \gamma^{2}}-\frac{1-\gamma}{\gamma^{2}} \lambda_{I} \sigma_{I}
\end{aligned}
$$

and $\hat{z}=\mathcal{C}^{-1} z$ with $\mathcal{C}$ lower-triangle Cholesky decomposition of $\mathcal{R}_{X X}\left(\mathcal{C C}^{\top}=\right.$ $\left.\mathcal{R}_{X X}\right)$.

(ii) Based on the solution in (i), the optimal portfolio can be expressed as

$$
\left(\begin{array}{c}
\alpha_{1 t} \\
\alpha_{2 t} \\
\alpha_{3 t} \\
\alpha_{4 t}
\end{array}\right)=\frac{1}{\gamma}\left(\Sigma_{t}^{\top}\right)^{-1} \mathcal{R}_{A A}^{-1}\left(\begin{array}{c}
\lambda_{r} \\
\lambda_{\pi} \\
\lambda_{I} \\
\lambda_{S}
\end{array}\right)+\left(1-\frac{1}{\gamma}\right)\left(\Sigma_{t}^{\top}\right)^{-1}\left(\begin{array}{c}
-g_{r} B_{r}(T-t) \\
0 \\
\sigma_{I} \\
0
\end{array}\right)
$$

\section{Remarks}

(i) Recall we have two factors $X_{t}=\left(r_{t}, \pi_{t}\right)$ while in our solution (33) only one factor, $r_{t}$, appears. This is due to the no-arbitrage equality (26). Inspection of the partial differential equation (32) reveals that only the second term in the fourth line is related to the factors $X_{r}$. Rewriting the no-arbitrage equality (26) as

$$
R_{t}-\pi_{t}=r_{t}-\lambda_{I} \sigma_{I}
$$


and applying it to the last term in the equation (32), then the factor, $\pi_{t}$, disappears in the partial differential equation due to the no-arbitrage relation. This result that the value function depends only on $r_{t}$ has already been obtained by Brennan and Xia (2002), however, they employ a different arbitrage condition based on the real pricing kernel.

(ii) The optimal portfolio (36) is independent of the level of the factor $r_{t}$. However, the stochastic of the factor $r_{t}$ still affects the optimal portfolio through the mean-reverting parameter $\kappa_{r}$ and the diffusion coefficient $g_{r}$ appearing in $\Sigma_{t}$.

\section{Backward Markov Chain Approximation Method}

The Markov chain approximation (MCA) method, see Kushner and Dupuis (1992) solves the continuous-time stochastic control problem by approximating the original controlled process by a finite-state controlled process. For our finitetime control problem we obtain a finite-state controlled process by discretising the time space, by approximating the Wiener process by symmetric random walks and by using state space grids. We develop a backward iteration scheme which solves the value function backwards iteratively. In the next section we demonstrate convergence on a particular numerical example.

\subsection{Backward iteration scheme}

For a finite-state process, actions take place only at discrete time points $\{k \Delta\}_{k=0,1, \cdots, N_{\Delta}}$. We choose $\Delta$ so that $N_{\Delta}:=1 / \Delta$ is a natural number. The transition of the factor $X_{t}$ in (5) is approximated by the Euler-Maruyama scheme and is denoted by

$$
X_{(k+1) \Delta}=X_{k \Delta}+F\left(X_{k \Delta}\right) \Delta+G\left(X_{k \Delta}\right) u_{k}^{X}:=\hat{X}\left(X_{k \Delta}\right),
$$

where $u_{k}^{X}$ are $n$-dimensional symmetric random walks $\left(\mathcal{P}\left(u_{k i}^{X}= \pm \sqrt{\Delta}\right)=\frac{1}{2}\right.$ for each component of $u_{k}^{X}$ ) with covariance $\mathcal{R}_{X X} \Delta$.

For the real wealth dynamics we also adopt the Euler-Maruyama approximation in (8) and obtain

$$
\begin{aligned}
\frac{v_{(k+1) \Delta}}{v_{k \Delta}}= & 1+\left(R\left(X_{t}\right)-\psi_{t}-\pi\left(X_{t}\right)+\sigma_{I}^{2}\right) \Delta \\
& +\alpha_{t}^{\top}\left(\mu\left(X_{t}, t\right)-R\left(X_{t}\right) \underline{\mathbf{1}}-\sigma_{I} \Sigma\left(X_{t}, t\right) \mathcal{R}_{I}\right) \Delta+\alpha_{t}^{\top} \Sigma\left(X_{t}, t\right) u_{k}-\sigma_{I} u_{k}^{I},
\end{aligned}
$$

where $t=k \Delta$. The correlations between $\left(u_{k}^{X}, u_{k}, u_{k}^{I}\right)$ are the same as for $\left(W_{t}^{X}, W_{t}, W_{t}^{I}\right)$. We note here that the proportional real wealth change is independent of the real wealth level $v_{t}$ and the price index $I_{t}$. Let $\hat{v}$ denote the real wealth transition mechanism from $t$ to $t+\Delta$. From (38) we know

$$
v_{t+\Delta}=: \hat{v}\left(v_{t}, X_{t}, t, \psi_{t}, \alpha_{t}\right) \text {, with } t=k \Delta \text {. }
$$


The consumption and portfolio decisions are only revised at the discrete-time points and remain constant over $[k \Delta,(k+1) \Delta)$, therefore the objective function of the given finite-state process can be written as

$$
\max _{\substack{c_{k \Delta}, \alpha_{k \Delta}, k=1, \cdots, T N_{\Delta}-1}} \mathbf{E}_{0}\left[\epsilon_{1} \sum_{k=0}^{T N_{\Delta}-1} e^{-\delta k \Delta} U\left(c_{k \Delta}\right)+e^{-\delta T} U\left(v_{T}\right)\right]
$$

with $c_{k \Delta}=\psi_{k \Delta} v_{k \Delta}$.

For our backward iteration scheme, let $J^{\Delta}\left(k \Delta, T, v_{k \Delta}, X_{k \Delta}\right)$ be the value function on the subperiod $[k \Delta, T]$ given the state $X_{k \Delta}$ and the real wealth $v_{k \Delta}$,

$$
J^{\Delta}\left(k \Delta, T, v_{k \Delta}, X_{k \Delta}\right)=\max _{\substack{c_{k^{\prime} \Delta}, \alpha_{k^{\prime} \Delta}, k^{\prime}=k, \cdots, T N_{\Delta}-1}} \mathbf{E}_{k \Delta}\left[\epsilon_{1} \sum_{k^{\prime}=k}^{T N_{\Delta}-1} e^{-\delta k^{\prime} \Delta} U\left(c_{k^{\prime} \Delta}\right)+e^{-\delta T} U\left(v_{T}\right)\right]
$$

where $k$ is any number from $\left\{0,1, \cdots, T N_{\Delta}-1\right\}$.

With simple algebraic rearrangement, we obtain the following backward iteration scheme:

Proposition 4 The stochastic control problem (40) with the transition dynamics (37) and (39) can be solved iteratively through the following formula;

$$
\begin{aligned}
& J^{\Delta}\left(k \Delta, T, v_{k \Delta}, X_{k \Delta}\right) \\
= & \max _{c_{k \Delta}, \alpha_{k} \Delta}\left\{\epsilon_{1} e^{-\delta(k \Delta)} U\left(c_{k \Delta}\right) \Delta+\mathbf{E}_{k \Delta}\left[J^{\Delta}\left((k+1) \Delta, T, v_{(k+1) \Delta}, X_{(k+1) \Delta}\right)\right]\right. \\
= & \max _{c_{k \Delta}, \alpha_{k \Delta}}\left\{\epsilon_{1} e^{-\delta(k \Delta)} U\left(c_{k \Delta}\right) \Delta\right. \\
& \left.\quad+\mathbf{E}_{k \Delta}\left[J^{\Delta}\left((k+1) \Delta, T, \hat{v}\left(v_{k \Delta}, X_{k \Delta}, k \Delta, \psi_{k \Delta}, \alpha_{k \Delta}\right), \hat{X}\left(X_{k \Delta}\right)\right)\right]\right\} .
\end{aligned}
$$

The iteration runs backwards from $t=(k+1) \Delta$ to $t=k \Delta$. The first iteration is indexed by $k=T N_{\Delta}-1$ and the initial value function is

$$
J^{\Delta}\left(T, T, v_{T}, X_{T}\right) \equiv e^{-\delta T} U\left(v_{T}\right)
$$

on the RHS of the iteration scheme (41). The solution of the stochastic problem (40) is obtained when $k$ goes to 0.

We can decompose the value function $J^{\Delta}$ further in a multiplicative form analogously to the decomposition of the continuous-time case in the equation (12).

Proposition 5 If, (i) the utility function is homothetic, and (ii) the growth rate of real wealth is independent of the real wealth level, then the discrete-time value function defined in (41) has the multiplicative form

$$
J^{\Delta}\left(k \Delta, T, v_{k \Delta}, X_{k \Delta}\right)=e^{-\delta k \Delta} U\left(v_{k \Delta}\right) \Theta^{\Delta}\left(k \Delta, T, X_{k \Delta}\right),
$$


for all $k=0,1, \cdots, T N_{\Delta}$, with

$$
\begin{aligned}
& \Theta^{\Delta}\left(k \Delta, T, X_{k \Delta}\right) \\
:= & (1-\gamma) \max _{\psi_{k \Delta}, \alpha_{k} \Delta}\left\{\epsilon_{1} U\left(\psi_{k \Delta}\right) \Delta\right. \\
& \left.+e^{-\delta \Delta} \mathbf{E}_{k \Delta}\left[U\left(\frac{\hat{v}}{v}\left(X_{k \Delta}, \psi_{k \Delta}, \alpha_{k \Delta}\right)\right) \Theta^{\Delta}\left((k+1) \Delta, T, \hat{X}\left(X_{k \Delta}\right)\right)\right]\right\},
\end{aligned}
$$

The iteration is defined through a backward scheme from $t=(k+1) \Delta$ to $t=k \Delta$. For the first iteration with $k=T N_{\Delta}-1$ we have

$$
\Theta^{\wedge}\left(T, T, X_{T}\right) \equiv 1
$$

on the RHS of the iteration scheme (44).

The product form above is similar with the product form (12) in the continuoustime model and both are based on the same reasoning. Due to this we can iterate $\Theta^{\Delta}$ without considering $v_{t}$, which greatly reduces the calculation cost.

To obtain a finite-state controlled process we still need to discretise the state space. We employ the truncation technique of Camilli and Falcone (1995), which truncates the control problem on a compact state space which is "large enough". Then, we take cuboidal grids on the chosen compact set and use the multilinear interpolation for the value function as described in Gruene (2001).

\subsection{Remarks on Convergence of the Algorithm}

Kushner and Dupuis (2001) give conditions under which the MCA method converges to its continuous-time solution as the time step and the grid size converge to zero, see P. 70-71, P276. Essentially these conditions are

C1. The approximating finite-state processes are "local consistent".

C2. The optimal control policy has a "relaxed control representation".

C3. The drift and diffusion coefficients of the state variables are bounded and continuous.

C4. The space of control variables is compact and the utility function $U$ is continuous and bounded

The local consistency C1 defined in P.71 Kushner and Dupuis (2001) requires that the approximating finite-state processes are close to the original process. It is automatically satisfied if we consider the Euler-Maruyama scheme. Condition $\mathrm{C} 2$ requires the optimal control policy to have some "nice" property such that it can be approximated by a piecewise constant and finite-valued control policy with arbitrary small penalty on the value function, see P.86-87 Kushner and 
Dupuis (2001). We will see this convergence later in the numerical examples. With regard to Condition $\mathrm{C} 3$ recall that the state variables are $\left(X_{t}, v_{t}\right)$. The variable $t$ satisfies $\mathrm{C} 3$ directly. For $X_{t}$ it is also satisfied using the truncated problem of Camilli and Falcone (1995) where $X_{t}$ is confined to a compact set. It is difficult to require $\mathrm{C} 3$ for the last state variable $v_{t}$ if we do not put any constraint on the portfolio decision $\alpha_{t}$. Then the agents are allowed to hold extreme positions which may cause extreme wealth movements. However, a rational agent will not take extreme positions but optimize her/his asset allocation according to the utility function. For our case where $\alpha_{t}$ can be solved analytically we know how to choose a compact set for $\alpha_{t}$ which includes all the maxima. Considering the stochastic control problem on this compact set the conditions $\mathrm{C} 3$ and $\mathrm{C} 4$ then can be satisfied. In the case of short-sale constraints, Conditions C3 and C4 are satisfied easily when we exclude short-sale possibilities.

\section{Numerical Study}

In the numerical study we investigate the performance of the backward MCA method with different time steps and grid sizes. Then, we apply it to find optimal strategies under several kinds of short-sale constraints. We use the software packages "GAUSS" with the two application packages, "Optimization" and "Constrained Optimization".

The parameters for the numerical study in Table 1 are adopted from the model calibration to US data, given by Hsiao, Chiarella and Semmler (2005).

Table 1: Parameters.

$$
\begin{array}{llll}
\rho_{r \pi}=-0.02 & \rho_{r I}=0.07 & \rho_{r S}=0.17 & \\
\rho_{\pi I}=-0.02 & \rho_{\pi S}=0.10 & \rho_{I S}=-0.06 & \\
\lambda_{r}=-0.13 & \lambda_{\pi}=-0.57 & \lambda_{I}=0.64 & \lambda_{S}=0.87 \\
g_{r}=0.01 & g_{\pi}=0.0064 & \sigma_{I}=0.01 & \sigma_{S}=0.14 \\
\kappa_{r}=3.00 & \bar{r}=0.0014 & \kappa_{\pi}=0.50 & \bar{\pi}=0.035
\end{array}
$$

We truncate our control problem on the compact set $-2 \% \leq r_{t} \leq 2 \%$. The invariant distribution of the process $r_{t}$ defined in (16) has standard deviation $0.004082\left(=g_{r}^{2} / 2 \kappa_{r}\right)$ and according to this distribution the probability of being out of this compact set is extremely low $\left(9.6 \cdot 10^{-7}\right)$. The investment horizon is set to be 5 years. The two nominal bonds for investment have 2 and 5 years maturity. When the first bond matures at the end of the second and the fourth years, another 2-year bond will be introduced immediately. The relative risk aversion parameter is set at $\gamma=4.0$. The subjective discount factor $\delta$ is chosen as $\delta=0.02$. We consider the case without intermediate consumption $\epsilon_{1}=0$. 
We solve this stochastic control problem with different time step $\Delta=0.5,0.05,0.005$ and different grid sizes $\Delta r=0.002,0.0002$. Table 2 gives average errors of the value function of the numerical solutions compared to the corresponding theoretical solution given in Proposition 3. Table 3 gives relative average errors of the optimal portfolio decisions with respect to the theoretical solution. The convergence criterion for the gradients in the numerical optimization was set at $10^{-8}$.

Table 2: Average Errors of the Value Function.

\begin{tabular}{lrr} 
& $\Delta_{\text {state }}=0.002$ & $\Delta_{\text {state }}=0.0002$ \\
\hline$\Delta_{\text {time }}=0.5$ & $-1012 \cdot 10^{-5}$ & $-1012 \cdot 10^{-5}$ \\
$\Delta_{\text {time }}=0.05$ & $-104 \cdot 10^{-5}$ & $-104 \cdot 10^{-5}$ \\
$\Delta_{\text {time }}=0.005$ & $-4 \cdot 10^{-5}$ & $-17 \cdot 10^{-5}$
\end{tabular}

Table 3: Average Relative Errors of the Portfolio Decisions.

\begin{tabular}{ccrr} 
& & $\Delta_{\text {state }}=0.002$ & $\Delta_{\text {state }}=0.0002$ \\
\hline$\Delta_{\text {time }}=0.5$ & $\alpha_{1}$ & $48 \%$ & $49 \%$ \\
& $\alpha_{2}$ & $11 \%$ & $12 \%$ \\
& $\alpha_{3}$ & $13 \%$ & $13 \%$ \\
& $\alpha_{4}$ & $47 \%$ & $47 \%$ \\
$\Delta_{\text {time }}=0.05$ & & & \\
& $\alpha_{1}$ & $1.72 \%$ & $1.80 \%$ \\
& $\alpha_{2}$ & $0.18 \%$ & $0.22 \%$ \\
& $\alpha_{3}$ & $0.87 \%$ & $0.86 \%$ \\
$\Delta_{\text {time }}=0.005$ & $\alpha_{4}$ & $3.74 \%$ & $2.80 \%$ \\
& $\alpha_{1}$ & $4.70 \%$ & \\
& $\alpha_{2}$ & $1.95 \%$ & $1.24 \%$ \\
& $\alpha_{3}$ & $0.10 \%$ & $0.56 \%$ \\
& $\alpha_{4}$ & $0.26 \%$ & $0.10 \%$ \\
& & & $0.26 \%$
\end{tabular}

We can see in Tables 2 and 3 that the performance of the numerical algorithm improves when the time step reduces while it does not improve much when the grid size decreases. When comparing between different time steps, by the refinement of the time step from 0.05 to 0.005 , Table 2 shows the fitting of the value function becomes better while the fitting of the optimal portfolios in Table 3 does not improve much. Considering the trade-off between numerical precision and calculation cost we have decided to choose the time step $\Delta_{\text {time }}=0.05$ and the grid size $\Delta_{\text {state }}=0.002$ and implement our numerical process to solve for investment strategies with short-sale constraints.

We consider three kinds of short-sale constraints: 
SSC-1. Short-sale commissions: investors have to pay an additional $\eta$ units of commission for each unit short position. In our example $\eta$ is equal to 0.0002 .

SSC-2. Short-sale exclusion for all risky assets

SSC-3. Short-sale exclusion for all assets including the money market account.

Table 4 gives the average investment proportions under the short-sale constraints. Recall from Property 3 that the theoretical values of $\alpha$ are independent of the state variable $r_{t}$.

Table 4: Effect of Short-Sale Constraints.

\begin{tabular}{lrrrr} 
& $\alpha_{1}$ & $\alpha_{2}$ & $\alpha_{3}$ & $\alpha_{4}$ \\
\hline Theoretical values & -13.86 & 23.74 & 18.63 & 1.86 \\
Numerical values & -13.62 & 23.70 & 18.79 & 1.93 \\
SSC-1 commissions & 0.00 & 13.93 & 18.29 & 1.92 \\
SSC-2 exclusion, risky & 6.32 & 9.53 & 18.29 & 1.93 \\
SSC-3 exclusion, all & 0.00 & 0.00 & 0.00 & 1.00
\end{tabular}

All short-sale constraints change significantly the decisions concerning the optimal portfolio positions. When the short-sale commission is introduced as in the example, we can see in the line "SSC-1 commissions" the agents do not purchase the short-term bond which the agents would short-sell if there were no commissions. If the short-sale possibility is excluded for the risky assets as shown in "SSC-2", the agents reduce their holding in the nominal bonds while keep their positions in the inflation-indexed bond and the stock. If now the short-sale possibility is excluded for all assets, our agents only wish to hold the stock.

To analyze further the effect of the commission fees on the portfolio decisions, we provide simulation results with different fees in Table 5. As expected, the higher the commission fee, the smaller is the short position $\alpha_{1}$ (of the short-run bond). We observe also that large positions in the long-run bond $\alpha_{2}$ decrease with the commission fees. Whereas the positions of the inflation-indexed bonds and the stock are not changed by the introduction of the commission fees.

Table 5: Effect of Commission Fees on Portfolio Decisions

\begin{tabular}{lrrrr} 
comm. fees & $\alpha_{1}$ & $\alpha_{2}$ & $\alpha_{3}$ & $\alpha_{4}$ \\
\hline$\eta=0$ & -13.62 & 23.70 & 18.79 & 1.93 \\
$\eta=0.0001$ & -5.65 & 18.00 & 18.52 & 1.92 \\
$\eta=0.0002$ & 0.00 & 13.93 & 18.29 & 1.92
\end{tabular}


We analyze the SSC-3 portfolio decision further. In our numerical example the stock has a slightly higher market price of risk $\lambda_{S}=0.87$ and a significantly higher volatility $\sigma_{S}=0.14$ in comparison with the other volatilities $g_{r}, g_{\pi}$ in Table 1. Therefore, the stock has a relatively higher excess return $\lambda_{S} \sigma_{S}$ and also a quite higher risk $\sigma_{S}$ as given in (27). So, a risk-friendly investor would wish to invest in the stock. Given this consideration we would expect the stock holding of a risk averse agent would be smaller. We increase the agents' risk aversion from $\gamma=4$ to $\gamma=15$ and $\gamma=45$. The optimal strategies, both with and without the short-sale exclusion, are given in Table 6 . Under short-sale exclusion for all assets, the agents increase their holding of the inflation-indexed bonds, due to the fact that it is considered as a hedging asset, and decrease their stock holding.

Table 6: Risk Aversion and Short-Sale Exclusion

\begin{tabular}{llrrrr} 
Risk Aversion & SSC-3 & $\alpha_{1}$ & $\alpha_{2}$ & $\alpha_{3}$ & $\alpha_{4}$ \\
\hline$\gamma=4$ & without & -13.86 & 23.74 & 18.63 & 1.86 \\
$\gamma=15$ & without & -3.69 & 6.33 & 5.70 & 0.50 \\
$\gamma=45$ & without & -1.23 & 2.11 & 2.57 & 0.17 \\
& & & & & \\
$\gamma=4$ & with & 0.00 & 0.00 & 0.00 & 1.00 \\
$\gamma=15$ & with & 0.00 & 0.09 & 0.52 & 0.39 \\
$\gamma=45$ & with & 0.00 & 0.05 & 0.82 & 0.13
\end{tabular}

\section{Conclusion}

This paper solves the decision problem of intertemporal portfolios under inflation risk. Several short-sale constraints are considered and the optimal intertemporal portfolios under short-sale constraints are solved by means of a numerical method - the backward MCA (Markov chain approximation) method. In the case without short-sale constraints we have an analytical solution. Using this knowledge we can choose an "optimal" discretization of time and state spaces, with a view to both precision and numerical cost for implementing the numerical method. We find that all three short-sale constraints have a significant impact on the intertemporal portfolio decisions.

\section{Appendix}

Expression of $A_{n}(\tau), B_{n r}(\tau)$ and $B_{n \pi}(\tau)$ for the Duffie and Kan family. Applying Itô's Lemma on the Duffie-Kan bond price (18), we obtain the bond return as

$$
\frac{d P_{n}\left(r_{t}, \pi_{t}, t, T\right)}{P_{n}\left(r_{t}, \pi_{t}, t, T\right)}=\mu_{n}(t, T-t) d t-B_{n r}(T-t) g_{r} d W_{t}^{r}-B_{n \pi}(T-t) g_{\pi} d W_{t}^{\pi},
$$


where

$$
\begin{aligned}
\mu_{n}(t, \tau)= & A_{n}(\tau)+B_{n r}(\tau) r_{t}+B_{n \pi}(\tau) \pi_{t} \\
& -B_{n r}(\tau) \kappa_{r}\left(\bar{r}-r_{t}\right)-B_{n \pi}(\tau) \kappa_{\pi}\left(\bar{\pi}-\pi_{t}\right) \\
& +\frac{1}{2}\left(B_{n r}(\tau)^{2} g_{r}^{2}+2 B_{n r}(\tau) B_{n \pi}(\tau) \sigma_{r} \sigma_{\pi} \rho_{r \pi}+B_{n \pi}(\tau)^{2} g_{\pi}^{2}\right) .
\end{aligned}
$$

The bond return was assumed to satisfy the no-arbitrage constraint (23) in Property 2 with $B_{n r}$ and $B_{n \pi}$ specified in the equations (24) and (25).

Replacing $R_{t}$ in the no-arbitrage equality (23) with the expression (19), this turns out a differential equation for $A_{n}(\tau)$. With a straightforward calculation we can verify that $A_{n}(\tau)$ is solved as

$$
\begin{aligned}
\frac{A_{n}(\tau)}{\tau}= & \left(1-\frac{1}{\tau \kappa_{r}}+\frac{e^{-\tau \kappa_{r}}}{\tau \kappa_{r}}\right)\left(\bar{r}-\frac{g_{r} \lambda_{r}}{\kappa_{r}}\right)+\left(1-\frac{1}{\tau \kappa_{\pi}}+\frac{e^{-\tau \kappa_{\pi}}}{\tau \kappa_{\pi}}\right)\left(\bar{\pi}-\frac{g_{\pi} \lambda_{\pi}}{\kappa_{\pi}}\right) \\
& -\frac{g_{r}^{2}}{2 \kappa_{r}^{2}}\left(1-2 \frac{1-e^{-\kappa_{r} \tau}}{\kappa_{r} \tau}+\frac{1-e^{-2 \kappa_{r} \tau}}{2 \kappa_{r} \tau}\right)-\frac{g_{\pi}^{2}}{2 \kappa_{\pi}^{2}}\left(1-2 \frac{1-e^{-\kappa_{\pi} \tau}}{\kappa_{\pi} \tau}+\frac{1-e^{-2 \kappa_{\pi} \tau}}{2 \kappa_{\pi} \tau}\right) \\
& -\frac{\sigma_{r \pi}}{\kappa_{r} \kappa_{\pi}}\left(1-\frac{1-e^{-\kappa_{r} \tau}}{\kappa_{r} \tau}-\frac{1-e^{-\kappa_{\pi} \tau}}{\kappa_{\pi} \tau}+\frac{1-e^{-\left(\kappa_{r}+\kappa_{\pi}\right) \tau}}{\left(\kappa_{r}+\kappa_{\pi}\right) \tau}\right)+\xi_{0}
\end{aligned}
$$

with $\xi_{0}=A(0)$.

Proof of Proposition 3.

From the Property 7 in Hsiao, Chiarella and Semmler (2005), $\Phi\left(t, T, X_{t}\right)$ is given by

$$
\Phi\left(t, T, r_{t}\right)=\mathbf{E}_{t, x}\left[\exp \left(\frac{1-\gamma}{\gamma} \int_{t}^{T} r_{s} d s+h(T-t) d s\right) \frac{d \hat{\mathcal{P}}_{T \mid t}}{d \mathcal{P}_{T \mid t}}\right]
$$

where

$$
\begin{aligned}
h= & -\frac{\delta}{\gamma}+\frac{1-\gamma}{\gamma}\left(\sigma_{I}^{2}-\lambda_{I} \sigma_{I}\right)+\frac{1-\gamma}{2 \gamma^{2}} \lambda^{\top} \mathcal{R}_{A A}^{-1} \lambda \\
& +\frac{(1-\gamma)^{3}}{2 \gamma^{2}} \sigma_{I}^{2} \mathcal{R}_{I A} \mathcal{R}_{A A}^{-1} \mathcal{R}_{A I}-\frac{(1-\gamma)^{2}}{\gamma^{2}} \lambda^{\top} \mathcal{R}_{A A}^{-1} \mathcal{R}_{A I} \sigma_{I}-\frac{1-\gamma}{2} \sigma_{I}^{2} \\
\frac{d \hat{\mathcal{P}}_{T \mid t}}{d \mathcal{P}_{T \mid t}=} & \exp \left(z^{\top} \mathcal{R}_{X X}^{-1}\left(W_{T}^{X}-W_{t}^{X}\right)-\frac{1}{2} z^{\top} \mathcal{R}_{X X}^{-1} z(T-t)\right)
\end{aligned}
$$

with

$$
z=\frac{1-\gamma}{\gamma} \mathcal{R}_{X A} \mathcal{R}_{A A}^{-1} \lambda-\frac{(1-\gamma)^{2}}{\gamma} \mathcal{R}_{X A} \mathcal{R}_{A A}^{-1} \mathcal{R}_{A I} \sigma_{I}-(1-\gamma) \mathcal{R}_{X I} \sigma_{I}
$$

We note in (51) that $\mathcal{R}_{I A} \mathcal{R}_{A A}^{-1} \mathcal{R}_{A I}=1$ and $\lambda^{\top} \mathcal{R}_{A A}^{-1} \mathcal{R}_{A I} \sigma_{I}=\lambda_{I} \sigma_{I}$. Then, after some rearrangement we will obtain (35). In the expression for $z$ in (53) we have 


$$
\mathcal{R}_{X A} \mathcal{R}_{A A}^{-1} \lambda=\left(\begin{array}{c}
\lambda_{r} \\
\lambda_{\pi}
\end{array}\right)
$$

and

$$
\mathcal{R}_{X A} \mathcal{R}_{A A}^{-1} \mathcal{R}_{A I}=\mathcal{R}_{X I}=\left(\begin{array}{c}
\rho_{r I} \\
\rho_{\pi I}
\end{array}\right) .
$$

Using these two equalities above we will obtain (34).

The solution $r_{t}$ to the stochastic differential equation (16) is given by ${ }^{4}$

$$
r_{s}=e^{-\kappa_{r}(s-t)} r_{t}+\bar{r}\left(1-e^{-\kappa_{r}(s-t)}\right)+g_{r} \int_{t}^{s} e^{-\kappa_{r}(s-u)} d W_{u}^{r}
$$

Using this solution and Fubini's theorem, we get

$$
\begin{aligned}
\int_{t}^{T} r_{s} d s & =\left(r_{t}-\bar{r}\right) \int_{t}^{T} e^{-\kappa(s-t)} d s+\bar{r}(T-t)+g_{r} \int_{t}^{T} \int_{u}^{T} e^{-\kappa(s-u)} d s d W_{u}^{r} \\
& =B_{r}(t, T) r_{t}+\bar{r}\left(T-t-B_{r}(t, T)\right)+g_{r} \int_{t}^{T} B_{r}(u, T) d W_{u}^{r}
\end{aligned}
$$

where

$$
B_{r}(t, T)=\frac{1}{\kappa_{r}}\left(1-e^{-\kappa_{r}(T-t)}\right) .
$$

Using the notation $\mathcal{C C}^{\top}=\mathcal{R}_{X X}$ to rewrite (52) and letting

$$
\hat{z}=\mathcal{C}^{-1} z=\left(\begin{array}{l}
\hat{z}_{1} \\
\hat{z}_{2}
\end{array}\right), \quad \hat{W}_{t}^{X}=\mathcal{C}^{-1} W_{t}^{X}=\left(\begin{array}{l}
\hat{W}_{1 t}^{X} \\
\hat{W}_{1 t}^{X}
\end{array}\right)
$$

we have

$$
\frac{d \hat{\mathcal{P}}_{T \mid t}}{d \mathcal{P}_{T \mid t}}=\exp \left(\hat{z}^{\top}\left(\hat{W}_{T}^{X}-\hat{W}_{t}^{X}\right)-\frac{1}{2} \hat{z}^{\top} \hat{z}(T-t)\right) .
$$

Note that $\hat{W}_{t}^{X}$ is an orthogonal Wiener process because $\operatorname{Var}\left[\hat{W}_{1}^{X}\right]=\mathcal{C}^{-1} \mathcal{R}_{X X} \mathcal{C}^{-1 \top}=$ $I_{n}$.

Summarizing all the above calculations and letting

$$
\begin{aligned}
& \mathcal{Y}(t, T) \\
= & \frac{1-\gamma}{\gamma} B_{r}(T-t) r_{t}+\frac{1-\gamma}{\gamma} \bar{r}\left(T-t-B_{r}(T-t)\right)+h(T-t)-\frac{1}{2} \hat{z}^{\top} \hat{z}(T-t) \\
& +\int_{t}^{T}\left(\frac{1-\gamma}{\gamma} g_{r} B_{r}(T-u)+\hat{z}_{1}\right) d \hat{W}_{1 u}^{X}+\hat{z}_{2}\left(\hat{W}_{2 T}^{X}-\hat{W}_{1 t}^{X}\right),
\end{aligned}
$$

\footnotetext{
${ }^{4}$ See for example Kloeden and Platen (1992).
} 
then we can write

$$
\Phi\left(t, T, r_{t}\right)=\mathbf{E}_{t, x}[\exp \mathcal{Y}(t, T)] .
$$

Note that $\mathcal{Y}(t, T)$ is normally distributed with the mean and the variance given by

$$
\begin{aligned}
\mathbf{E}_{t, x}[\mathcal{Y}(t, T)] & =\frac{1-\gamma}{\gamma} B_{r}(T-t) r_{t}+\frac{1-\gamma}{\gamma} \bar{r}\left(T-t-B_{r}(T-t)\right)+h(T-t)-\frac{1}{2} \hat{z}^{\top} \hat{z}(T-t), \\
\operatorname{Var}_{t, x}[\mathcal{Y}(t, T)] & =\int_{t}^{T}\left(\frac{1-\gamma}{\gamma} g_{r} B_{r}(T-u)+\hat{z}_{1}\right)^{2} d u+\hat{z}_{2}^{2}(T-t) .
\end{aligned}
$$

Using the equality

$$
\mathbf{E}_{t, x}[\exp (\mathcal{Y}(t, T))]=\exp \left(\mathbf{E}_{t, x}[\mathcal{Y}(t, T)]+\frac{1}{2} \operatorname{Var}_{t, x}[\mathcal{Y}(t, T)]\right)
$$

we obtain the result (33).

Proof of Proposition 5.

We prove this property through mathematical induction.

We start with $k=T N_{\Delta}$. Following the definition $J^{\Delta}\left(T, T, v_{T}, X_{T}\right)=e^{-\delta T} U\left(v_{T}\right)$, the statement (43) is satisfied with $\Theta^{\wedge}\left(T, T, X_{t}\right) \equiv 1$.

Assume the multiplicative form (43) holds for $k+1$. Rewriting the iteration formula (41) we obtain

$$
\begin{aligned}
& J^{\Delta}\left(k \Delta, T, v_{k \Delta}, X_{k \Delta}\right) \\
& =\max _{c_{k \Delta}, \alpha_{k} \Delta}\left\{\epsilon_{1} e^{-\delta(k \Delta)} U\left(c_{k \Delta}\right) \Delta\right. \\
& \left.\quad+\mathbf{E}_{k \Delta}\left[e^{-\delta((k+1) \Delta)} U\left(v_{(k+1) \Delta}\right) \Theta^{\Delta}\left((k+1) \Delta, T, X_{(k+1) \Delta}\right)\right]\right\}, \\
& =e^{-\delta(k \Delta)}\left(v_{k \Delta}\right)^{1-\gamma} \max _{\psi_{k \Delta}, \alpha_{k} \Delta}\left\{\epsilon_{1} U\left(\psi_{k \Delta}\right) \Delta\right. \\
& \left.\quad+e^{-\delta \Delta} \mathbf{E}_{k \Delta}\left[U\left(\frac{\hat{v}}{v}\left(X_{k \Delta}, \psi_{k \Delta}, \alpha_{k \Delta}\right)\right) \Theta^{\Delta}\left((k+1) \Delta, T, \hat{X}\left(X_{k \Delta}\right)\right)\right]\right\},
\end{aligned}
$$

which establishes the result.

\section{References}

[1] M.J. Brennan and Y. Xia, Dynamic asset allocation under inflation, The Journal of Finance 57 (2002), no. 3, 1201-1238. 
[2] F. Camilli and M. Falcone, An approximation scheme for the optimal control of diffusion processes, Mathematical Modelling and Numerical Analysis 25 (1995), no. 1, 97-122.

[3] J.C. Cox and C-F. Huang, Optimal consumption and portfolio policies when asset prices follow a diffusion process, Journal of Economic Theory 49 (1989), 33-83.

[4] D. Duffie and R. Kan, A yield-factor model of interest rates, Mathematical Finance 6 (1996), no. 4, 397-406.

[5] L. Gruene, Numerik optimaler steuerung, Internet, http://www.math.unifrankfurt.de/ numerik/lehre/gruene/optctrl01/.

[6] C.-Y. Hsiao, C. Chiarella, and W. Semmler, Strategic asset allocation with investment in inflation-indexed bonds, Working Paper, University of Bielefeld, 2005.

[7] R. Jarrow and Y. Yildirim, Pricing treasury inflation protected securities and related derivatives using an HJM model, Journal of Financial and Quantitative Analysis 38 (2003), no. 2, 337-358.

[8] M. I. Kamien and N.L. Schwartz, Dynamic optimization, North-Holland, 1991.

[9] P. E. Kloeden and E. Platen, Numerical solution of stochastic differential equations, Springer, 1992.

[10] H.J. Kushner, Probability methods for approximations in stochastic control and for ellipic equations, Academic Press, New York, 1977.

[11] H.J. Kushner and P. Dupuis, Numerical methods for stochastic control problems in continuous time, second ed., Springer, 2001.

[12] J. Liu, Portfolio selection in stochastic environments, Stanford GSB Working Papers (2005).

[13] R.C. Merton, Optimum consumption and portfolio rules in a continuoustime model, Journal of Economic Theory 3 (1971), 373-413.

[14] - An intertemporal capital asset pricing model, Econometrica 41 (1973), no. 5, 867-887.

[15] B. Oksendal, Stochastic differential equations, 6th. ed., Springer, 2000.

[16] C.S Tapiero and A. Sulem, Computational aspects in applied stochastic control, Computational Economics 7 (1994), 109-146.

[17] J.A. Wachter, Portfolio and consumption decisions under mean-reverting returns: An exact solution for complete markets, Journal of Financial and Quantitative Analysis 37 (2002), no. 1, 63-91. 